\title{
Human Adipose-Derived Mesenchymal Stem Cells Ameliorate Elastase-Induced Emphysema in Mice by Mesenchymal-Epithelial Transition
}

\author{
Nobuhiro Fujioka' \\ Masahiro Kitabatake ${ }^{2}$ \\ Noriko Ouji-Sageshima ${ }^{2}$ \\ Takahiro Ibaraki ${ }^{\prime}$ \\ Makiko Kumamoto' \\ Yukio Fujita' \\ Shigeto Hontsu' \\ Motoo Yamauchi' \\ Masanori Yoshikawa' \\ Shigeo Muro (D) \\ Toshihiro Ito ${ }^{2}$ \\ 'Department of Respiratory Medicine, \\ Nara Medical University, Kashihara, Nara, \\ Japan; ${ }^{2}$ Department of Immunology, Nara \\ Medical University, Kashihara, Nara, \\ Japan
}

\begin{abstract}
Purpose: Chronic obstructive pulmonary disease (COPD) is a worldwide problem because of its high prevalence and mortality. However, there is no fundamental treatment to ameliorate their pathological change in COPD lung. Recently, adipose-derived mesenchymal stem cells (ADSCs) have attracted attention in the field of regenerative medicine to repair damaged organs. Moreover, their utility in treating respiratory diseases has been reported in some animal models. However, the detailed mechanism by which ADSCs improve chronic respiratory diseases, including COPD, remains to be elucidated. We examined whether human ADSCs (hADSCs) ameliorated elastase-induced emphysema and whether hADSCs differentiated into alveolar epithelial cells in a murine model of COPD.
\end{abstract}

Methods: Female SCID-beige mice (6 weeks old) were divided into the following four groups according to whether they received an intratracheal injection of phosphate-buffered saline or porcine pancreatic elastase, and whether they received an intravenous injection of saline or hADSCs 3 days after intratracheal injection; Control group, hADSC group, Elastase group, and Elastase-hADSC group. We evaluated the lung function, assessed histological changes, and compared gene expression between hADSCs isolated from the lung of ElastasehADSC group and naïve hADSCs 28 days after saline or elastase administration.

Results: hADSCs improved the pathogenesis of COPD, including the mean linear intercept and forced expiratory volume, in an elastase-induced emphysema model in mice. Furthermore, hADSCs were observed in the lungs of elastase-treated mice at 25 days after administration. These cells expressed genes related to mesenchymal-epithelial transition and surface markers of alveolar epithelial cells, such as TTF-1, $\beta$-catenin, and E-cadherin.

Conclusion: hADSCs have the potential to improve the pathogenesis of COPD by differentiating into alveolar epithelial cells by mesenchymal-epithelial transition.

Keywords: chronic obstructive pulmonary disease, mesenchymal-epithelial transition, adipose derived mesenchymal stem cell, pulmonary function test

\section{Introduction}

Chronic obstructive pulmonary disease (COPD) induces respiratory symptoms such as dyspnea, coughing, and sputum because of abnormalities in airways and alveoli caused by smoking, exposure to biomass fuels, and air pollution. ${ }^{1}$ It has been reported that about 3.2 million people died of COPD in 2015 and COPD ranked third among the global age-standardized death rates for both sexes. ${ }^{2,3}$ In particular, advanced COPD causes pathological changes such as emphysema in the lungs, which is a major pathological change. Current treatments for COPD, such as bronchodilators, theophylline, and corticosteroids, are effective to reduce dyspnea
Correspondence: Toshihiro Ito Department of Immunology, Nara Medical University, 840 Shijo-cho, Kashihara, Nara, 634-852I, Japan Tel +8I-744-22-305I

Fax +8I-744-29-7503

Email toshi-ito@naramed-u.ac.jp 
and prevent exacerbations, which have succeeded in improving quality of life to reduce mortality. However, there is no treatment that reduces or normalizes the pathological changes in the lungs of COPD patients, because these treatments cannot prevent disease progression. ${ }^{4}$

Recently, the efficacy of mesenchymal stem cells (MSCs) has been reported in rodent models of lung disease. $^{5-10}$ Moreover, therapeutic efficacy has been shown in models of emphysema established by cigarette smoke exposure and elastase administration. ${ }^{1-16}$ It has been suggested that the therapeutic effect of MSCs is mediated by their ability to differentiate into tissues, ${ }^{17}$ immunomodulatory functions, ${ }^{18}$ paracrine effects, ${ }^{14}$ and anti-apoptotic effects. ${ }^{19}$ Since bone marrow-derived MSCs (BM-MSCs) were first discovered, bone marrow has been considered to be the main source of MSCs for clinical applications. Subsequently, MSCs have been isolated from adipose tissue that is attracting attention as an alternative to bone marrow. Adipose-derived MSCs (ADSCs) can be isolated more easily and at considerably larger amounts than bone marrow. ${ }^{20}$ It has been reported that MSCs derived from mouse adipose tissue differentiate into alveolar epithelial cells and ameliorate lung parenchymal destruction caused by elastase-induced emphysema in mice. ${ }^{21}$ Moreover, it has been reported that systemic administration of non-HLA-matched allogeneic human MSCs is safe in patients with moderate-tosevere COPD, ${ }^{22}$ which suggests the potential of MSC therapy in COPD patients.

In this study, we investigated whether human ADSCs (hADSCs) improved lung functional and histological changes in an elastase-induced mouse model of emphysema. We also examined whether hADSCs migrated into emphysematous lungs and their differentiation characteristics.

\section{Methods}

\section{Ethics Statement}

All animal experiments were approved by The Animal Care and Use Committee at Nara Medical University (Nara, Japan). All methods were performed in accordance with the Policy on the Care and Use of Laboratory Animals, Nara Medical University. The experimental protocols were approved by the Ethics Review Committee for Animal Experimentation of Nara Medical University (approval number 12582).

\section{Elastase-Induced Emphysema Mice Model}

Female SCID-beige mice (6 weeks old) were purchased from Oriental Bio Service (Kyoto, Japan) and kept under specific pathogen-free conditions in the animal care facility of Nara Medical University. To establish the COPD model, mice were injected intratracheally (i.t.) with 1.25 $\mathrm{U}$ porcine pancreatic elastase (EC134, Elastase-High Purity, porcine, Elastin Products Company, Inc., Owensville, MO) in $50 \mu \mathrm{L}$ saline (Otsuka Pharmaceutical Co., Ltd., Tokushima, Japan) following anesthesia induced by pentobarbital (Nacalai Tesque Inc., Kyoto, Japan). Control mice were i.t. injected with $50 \mu \mathrm{L}$ saline. Three days after injection, $1 \times 10^{6}$ hADSCs (Lonza, Basel, Switzerland) in $200 \mu \mathrm{L}$ phosphate-buffered saline (PBS; Wako, Osaka, Japan) were administered intravenously (i.v.). Control mice were i.v. administered $200 \mu \mathrm{L}$ PBS. In some experiments, $1 \times 10^{6} \mathrm{hADSCs}$ were labeled with PKH26 (Sigma-Aldrich Inc., St. Louis, MO) in accordance with manufacturer's protocol. Mice were divided into four groups as follows: (i) Control group (Saline i.t. + PBS i.v.), (ii) hADSC group (Saline i.t. + hADSCs i.v.), (iii) Elastase group (Elastase i.t. + PBS i.v.), and (iv) Elastase-hADSC group (Elastase i.t. + hADSCs i.v.).

\section{Evaluation of Lung Functions}

After the mice were anesthetized with $0.1 \mathrm{mg} / \mathrm{g}$ pentobarbital by intraperitoneal injection, tracheal intubation was performed with a 19-G catheter, followed by measurement of lung functions, such as forced vital capacity (FVC), forced expiratory volume and flow at $0.1 \mathrm{~s}\left(\mathrm{FEV}_{0.1}\right.$, $\mathrm{FEF}_{0.1}$ ), static compliance (Cst), peak expiratory flow $(\mathrm{PEF})$, Newtonian resistance (Rn), tissue damping (G), tissue elastance $(\mathrm{H})$, inspiratory capacity (IC), and respiratory system resistance $(\mathrm{Rrs})$ using a flexiVent ${ }^{\circledR}$ ventilation system (SCIREQ Inc., Montreal, Canada) as described previously. $^{23}$

\section{Histology}

After evaluating lung functions, mice were euthanized by blood collection from the left ventricle of the heart and then their lungs were collected for analysis. The left lobe of the lung was inflated by injecting $4 \%$ paraformaldehyde into the trachea at a constant pressure of $25 \mathrm{~cm} \mathrm{H}_{2} \mathrm{O}$ for 20 min and fixed overnight at room temperature. Fixed lungs were embedded in paraffin, cut into $2-\mu \mathrm{m}$-thick sections, and then stained with Mayer haematoxylin (Sakura 
Finetek Japan Co. Ltd., Tokyo, Japan) and eosin (Nacalai Tesque, Inc.).

\section{Immunofluorescence Staining of the Lung}

Left lungs were removed, embedded in optimum cutting temperature compound (Sakura Finetek Japan Co. Ltd.), and frozen in liquid nitrogen. Sections were cut at $5 \mu \mathrm{m}$ thicknesses and fixed in PBS with 4\% paraformaldehyde for $10 \mathrm{~min}$ at room temperature. After washing with PBS, the sections were placed in blocking solution that contained 10\% normal goat serum in PBS at room temperature, incubated in PBS for $1 \mathrm{hr}$, and then incubated overnight at $4{ }^{\circ} \mathrm{C}$ in a primary antibody solution. After washing three times with PBS, the sections were incubated with the secondary antibody for $1 \mathrm{hr}$ at room temperature. Nuclei were counterstained with DAPI $(2 \mu \mathrm{g} / \mathrm{mL}$, Dojindo Laboratories, Kumamoto, Japan). We used the following primary antibodies: rabbit anti-TTF-1 antibody (1:200; Abcam, Cambridge, UK; ab76013), rabbit anti- $\beta$-Catenin antibody (1:100; Cell Signaling Technology Inc., MA; \#8480), rabbit anti-pro-SP-C antibody (1:100; Abcam; ab170699), and rabbit anti-CDH1 (E-cadherin) antibody (1:100; Thermo Fisher Scientific, Waltham, MA; \#143249). Staining was visualized by Alexa Fluor 488 goat anti-rabbit IgG (1:400; Thermo Fisher Scientific; A32731) as the secondary antibody. Finally, the sections were analyzed under an FV1000 confocal microscope (Olympus, Tokyo, Japan).

\section{Lung PKH26-Labeled Cell Isolation}

To prepare a single cell suspension from the right lung, the lungs were dissociated using a Lung Dissociation Kit (Miltenyi Biotec, Cologne, Germany) with a gentle MACS Dissociator. The suspension was filtered through a $70 \mu \mathrm{m}$ cell strainer and the cells were collected by centrifugation following the manufacturer's instructions. After red blood cells were lysed with BD Pharm Lyse ${ }^{\mathrm{TM}}$ (BD Biosciences, Franklin Lakes, NJ), the cells were centrifuged $(400 \times \mathrm{g})$ and collected. PBS containing $0.5 \%$ bovine serum albumin (Wako) and $2 \mathrm{mM}$ EDTA (Wako) was added to the cells and PKH26-labeled cells were sorted using a FACSAria ${ }^{\mathrm{TM}}$ IIu Cell Sorter (BD Biosciences). Sorted PKH26-positive cells were collected in RLT buffer (Qiagen, Hilden, Germany) for RNA extraction.

\section{RNA Extraction and PCR Array}

Total RNA was extracted using a RNeasy Micro Kit (Qiagen) following the manufacturer's instructions. The amount of extracted RNA was measured using a NanoDrop ${ }^{\mathrm{TM}}$ (Thermo Fisher Scientific). The gene expression of hADSCs and sorted PKH26-positive cells from mouse lungs were analyzed using a $\mathrm{RT}^{2}$ Profiler $^{\mathrm{TM}}$ PCR Array Human Epithelial to Mesenchymal Transition (Qiagen). RNA was reverse transcribed into cDNA by incubation with reverse transcriptase at $42^{\circ} \mathrm{C}$ for $15 \mathrm{~min}$, followed by incubation at $95^{\circ} \mathrm{C}$ for $5 \mathrm{~min}$ using an $\mathrm{RT}^{2}$ First Strand Kit (Qiagen). cDNA was amplified with $\mathrm{RT}^{2}$ SYBR Green ROX qPCR Mastermix (Qiagen) in accordance with the manufacturer's instructions. Gene expression was normalized to multiple housekeeping genes. The results were analyzed using $\mathrm{RT}^{2}$ Profiler PCR Array Data Analysis v3.5 (Qiagen) by comparing gene expression between hADSCs and sorted hADSCs from the lungs of the Elastase-hADSC group.

\section{Statistical Analysis}

Statistical differences were analyzed by one-way analysis of variance with Tukey's multiple comparison test and the Student's $t$-test. A P-value of $<0.05$ was considered significant. Graphs and statistical tests were made using GraphPad Prism version 7.00 (GraphPad Software, San Diego, CA).

\section{Results}

\section{hADSC Administration Improves the Pathogenesis of Lung Emphysema in Mice}

We administered elastase or saline intratracheally to SCID-beige mice, followed 3 days later by intravenous administration of PBS or hADSCs. At 28 days after elastase administration, mice were euthanized after pulmonary function tests and their lungs were collected (Figure 1A). Lung histology in the Control group (Saline i.t. + PBS i.v.) did not show histological changes and damages, and that in the Elastase group (Elastase i.t. + PBS i.v.) showed air space enlargement and destruction of the alveolar walls compared with the Control group. However, in the Elastase-hADSC group (Elastase i.t. + hADSCs i.v.), the destruction of alveolar walls and the enlargement of alveolar spaces were milder than in the Elastase group (Figure 1B). The mean linear intercept (Lm) method, which was used to evaluate the emphysematous change, ${ }^{24}$ showed a significant change in the Elastase group (Lm: 20.5-34.5 $\mu \mathrm{m}$ ) compared with the Control group (Lm: 14.5-19.0 $\mu \mathrm{m}$ ) and significant reduction in the Elastase-hADSC group (Lm: 17.1- 
A

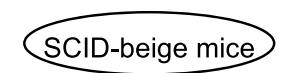

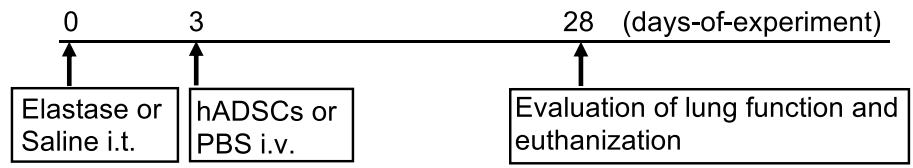

B

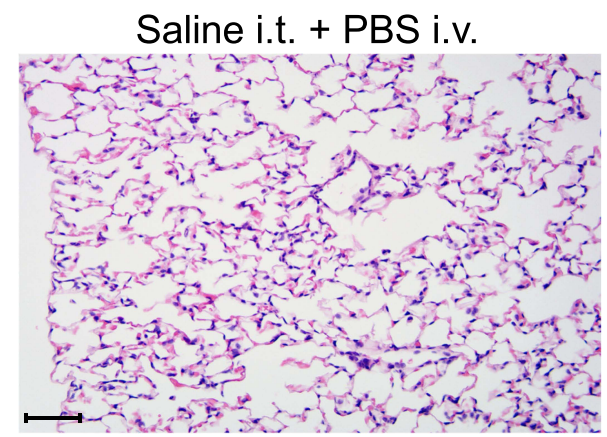
Elastase i.t. + PBS i.v.
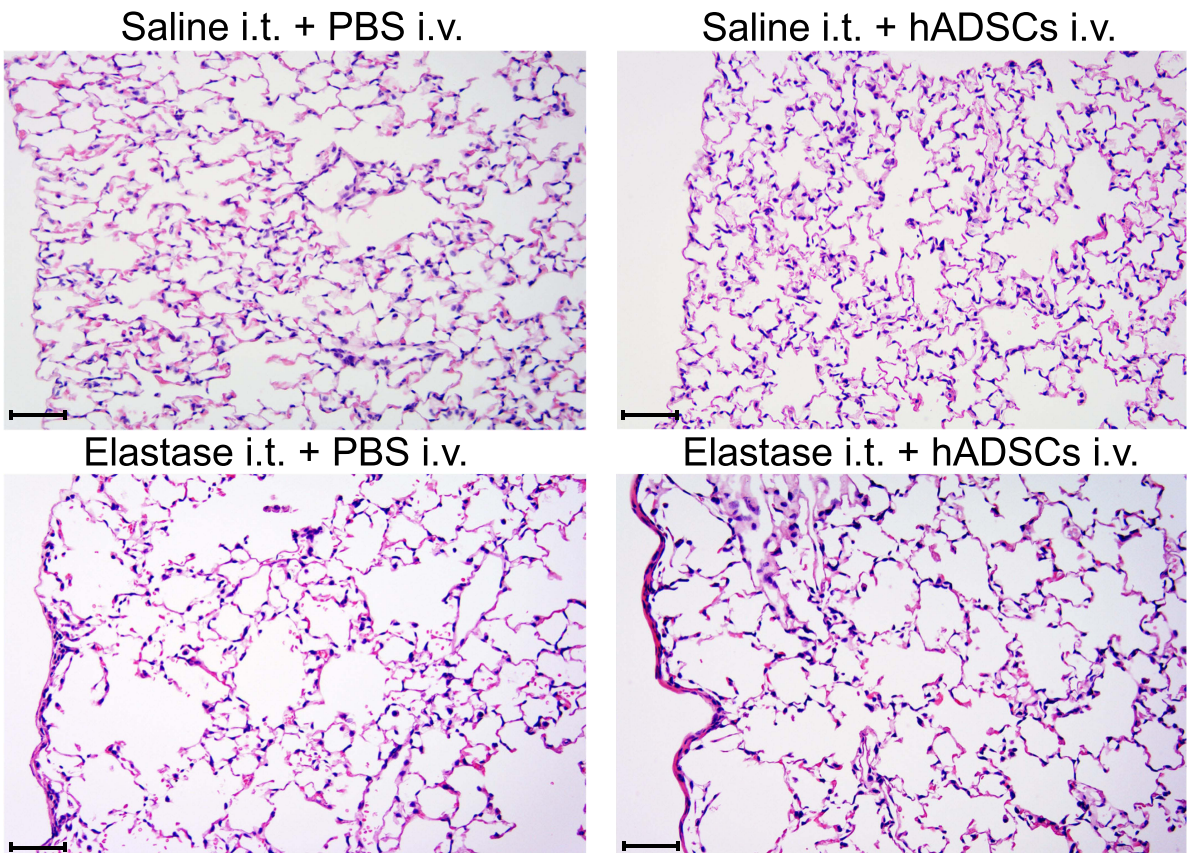

Saline i.t. + hADSCs i.v.
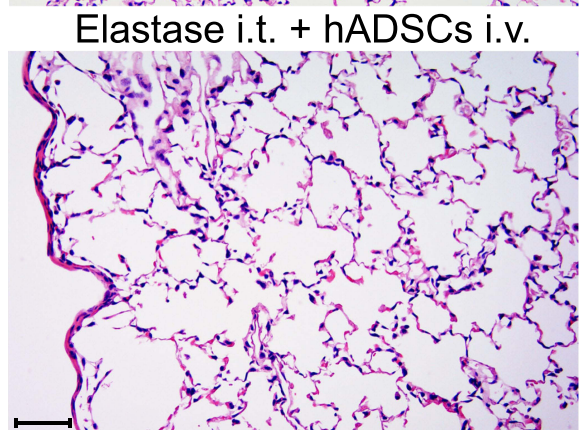

C

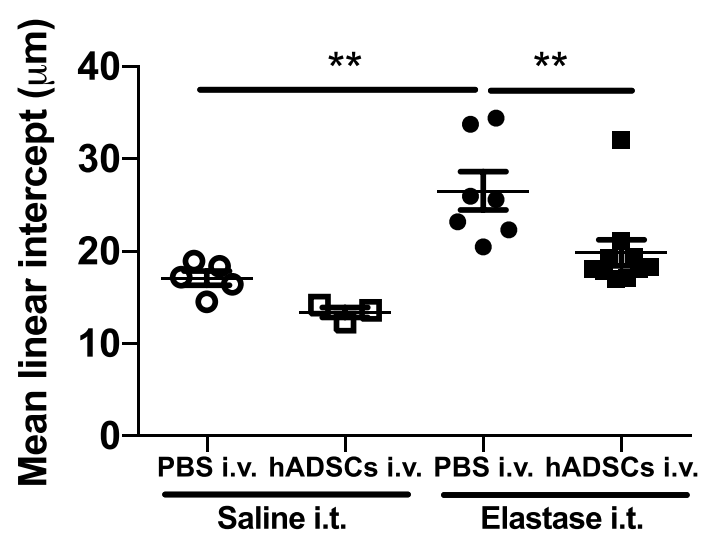

Figure I hADSCs suppress elastase-mediated lung histological changes in SCID-beige mice.

Notes: (A) Schematic representation of the experimental protocol. SCID-beige mice were intratracheally injected with saline or I.25 Uelastase and then intravenously injected with PBS or $1 \times 10^{6} \mathrm{hADSC}$ s onday 3 . Onday 28 , lungs were collected for analyses. (B) Lung histopathological analysis by hematoxylin and eosin staining $(\times 200)$. Scale bar $=50 \mu \mathrm{m}$. (C) Mean linear intercept of the four groups. Values are means \pm SEM. (i) Control group (Saline i.t. + PBS i.v.) ( $n=5$ ), (ii) hADSC group (Saline i.t. + hADSCs i.v.) $(n=3)$, (iii) Elastase group (Elastase i.t. + PBS i.v.) $(n=7)$, and (iv) Elastase-hADSC group (Elastase i.t. + hADSCs i.v.) $(n=10)$. **P < 0.01 .

Abbreviations: hADSCs, human adipose-derived mesenchymal stem cells; SEM, standard error of the mean; PBS, phosphate-buffered saline; i.t., intratracheally; i.v., intravenously.

$32.1 \mu \mathrm{m})$ compared with the Elastase group. However, Lm showed no significant change between the hADSC group (Saline i.t. + hADSCs i.v.) (Lm: 12.4-14.2 $\mu \mathrm{m}$ ) and the Control group (Figure 1C).

\section{hADSC Administration Improves the Lung Functions of Emphysema in Mice}

To evaluate the effect of hADSC administration, we performed a pulmonary function test. $\mathrm{FEV}_{0.1}$ was 


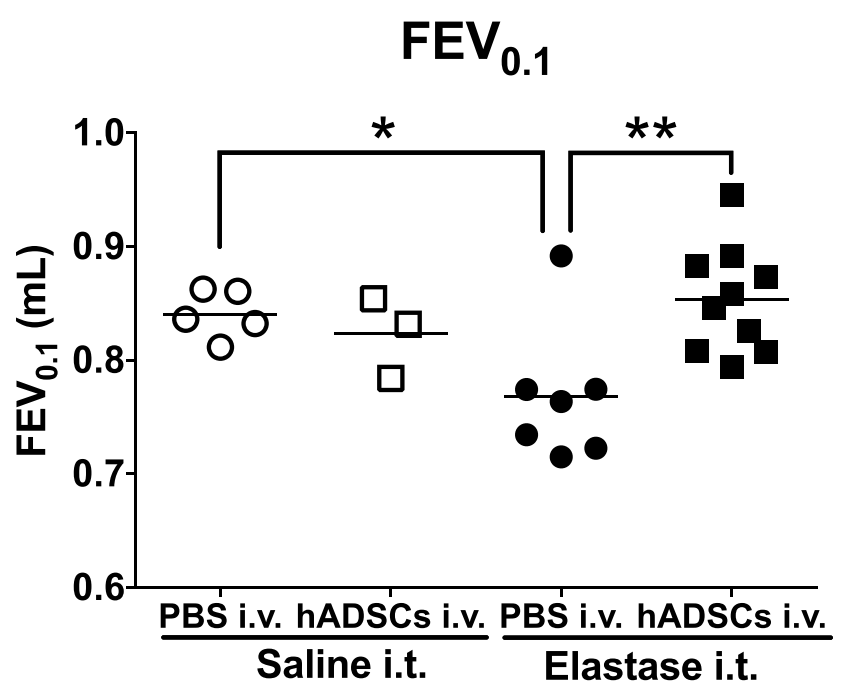

Figure 2 Lung function of emphysema mice treated with hADSCs.

Notes: The lung function $\mathrm{FEV}_{0.1}$ was measured using the flexiVent ${ }^{\circledR}$ ventilation system. Control group (Saline i.t. + PBS i.v.) $(n=5)$, hADSC group (Saline i.t. + hADSCs i.v.) $(n=3)$, Elastase group (Elastase i.t. + PBS i.v.) $(n=7)$, and ElastasehADSC group (Elastase i.t. + hADSCs i.v.) $(n=10)$. Values are presented as means \pm SEM. $* \mathrm{P}<0.05, * * \mathrm{P}<0.01$

Abbreviations: hADSCs, human adipose-derived mesenchymal stem cells; $\mathrm{FEV}_{0.1}$, forced expiratory volume at $0.1 \mathrm{~s}$; SEM, standard error of the mean.

significantly lower in the Elastase group than in the Control group and $\mathrm{FEV}_{0.1}$ was markedly improved in the Elastase-hADSC group $(0.8534 \pm 0.0148 \mathrm{~mL})$ compared with the Elastase group $(0.7682 \pm 0.0226 \mathrm{~mL})$ (Figure 2). However, there were no significant differences in other pulmonary function parameters including $\mathrm{FVC}, \mathrm{FEV}_{0.1}$ /FVC, Cst, FEF 0.1 , PEF, Rn, G, H, IC, and Rrs (Figure S1).

\section{hADSCs That Migrate to the Emphysematous Lung Express Genes Related to Mesenchymal-to-Epithelial Transition}

Next, we investigated how hADSCs had changed their phenotype after migration into emphysematous lungs. PKH26-labeled hADSCs were identified at around $1 \%$ of whole lung cells in the Elastase-hADSC group at day 28 (Figure 3A). We sorted PKH26-labeled hADSCs and compared gene expression between naïve hADSCs directly thawed from frozen stock and hADSCs sorted from the lungs of the Elastase-hADSC group using a $\mathrm{RT}^{2}$ profiler $^{\mathrm{TM}}$ PCR array Human Epithelial to Mesenchymal Transition. Gene expression of epithelial markers, such as Cdh1, Ctnnb1, Dsc2, Krt19, and Spp1, was higher in hADSCs from emphysematous lungs compared with naïve hADSCs. However, gene expression of mesenchymal markers, such as Cdh2, Col1a2, Col3a1, Fn1, Snai2, Twist1, Vim, Wnt5a, Wnt5b, and Zeb1, was lower in hADSCs from emphysematous lungs compared with naïve hADSCs (Figure 3B). These results suggest that hADSCs migrated into the emphysematous lungs and changed their phenotype by mesenchymal-to-epithelial transition.

\section{hADSCs That Migrate to the Emphysematous Lung Express Pulmonary Epithelial Surface Markers}

To define where hADSCs had migrated into the lungs, we next performed immunofluorescence staining of the lungs. We found very few PKH26-positive cells in the hADSC group, whereas PKH26-positive cells were sufficiently found in the Elastase-hADSC group (Figure 4A). Next, lung sections were stained with several alveolar epithelial markers, including TTF-1, Pro SP-C, $\beta$-Catenin, and CDH1 (E-cadherin) to investigate the characteristics of PKH-positive hADSCs in the lungs of the ElastasehADSC group. PKH-positive hADSCs were merged with TTF-1, $\beta$-Catenin, and CDH1, but negative for Pro SP-C, a type II epithelial marker (Figures 4B, $\underline{\mathrm{S} 2}$ and $\underline{\mathrm{S} 3})^{25}$ These data indicate that hADSCs might differentiate into type I alveolar epithelial cells in emphysematous lungs.

\section{Discussion}

In this study, we demonstrated that administration of hADSCs, which were migrated into emphysematous lungs, improved the pathogenesis and pulmonary function of emphysematous lungs in mice. Moreover, hADSCs attracted to the emphysematous lungs showed increased epithelial gene expression and decreased mesenchymal gene expression compared with naïve hADSCs, which indicated that hADSCs may have acquired the characteristics of type I alveolar epithelial cells by mesenchymalepithelial transition.

ADSCs are less invasive to collect than BM-MSCs, ${ }^{26}$ collected at larger numbers, ${ }^{27,28}$ and more effective suppressors of immune responses. ${ }^{20}$ Furthermore, hADSCs have been reported to be genetically and morphologically more stable in long-term culture, exhibit lower senescence rates and higher proliferative capacity, and retain differentiation potential for longer periods in culture than human BM-MSCs, ${ }^{29}$ suggesting that hADSCs are more suitable for clinical application among the MSC types. In fact, many preclinical studies using rodent models of 
A

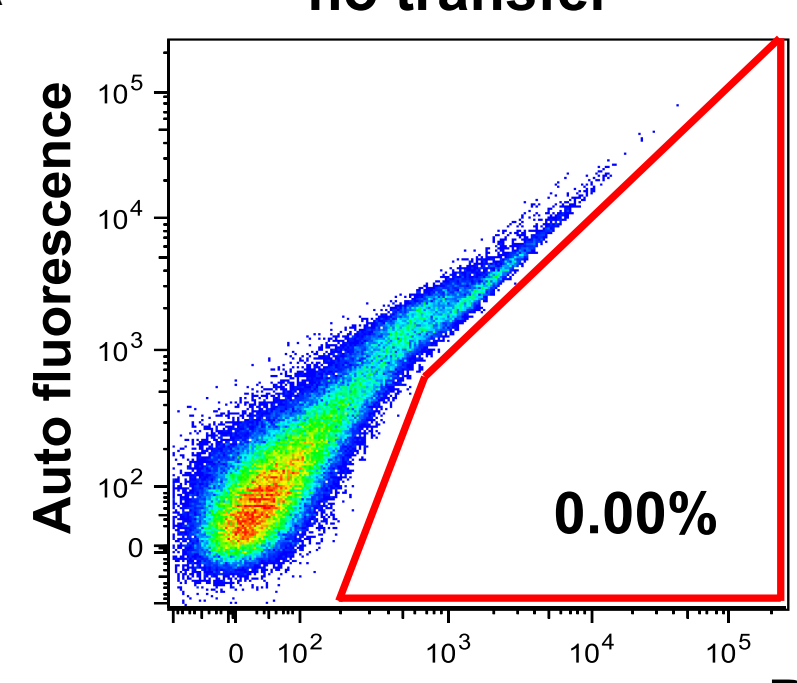

hADSCs transfer

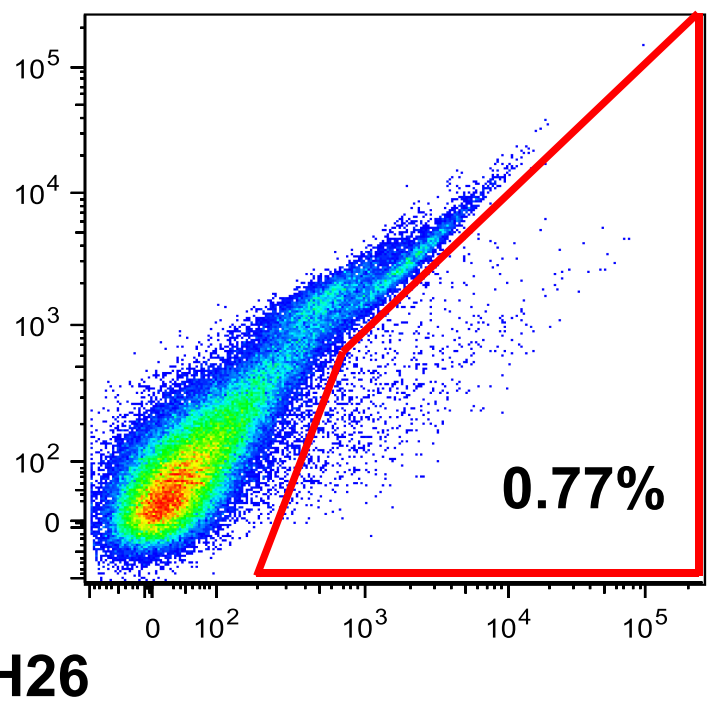

PKH26

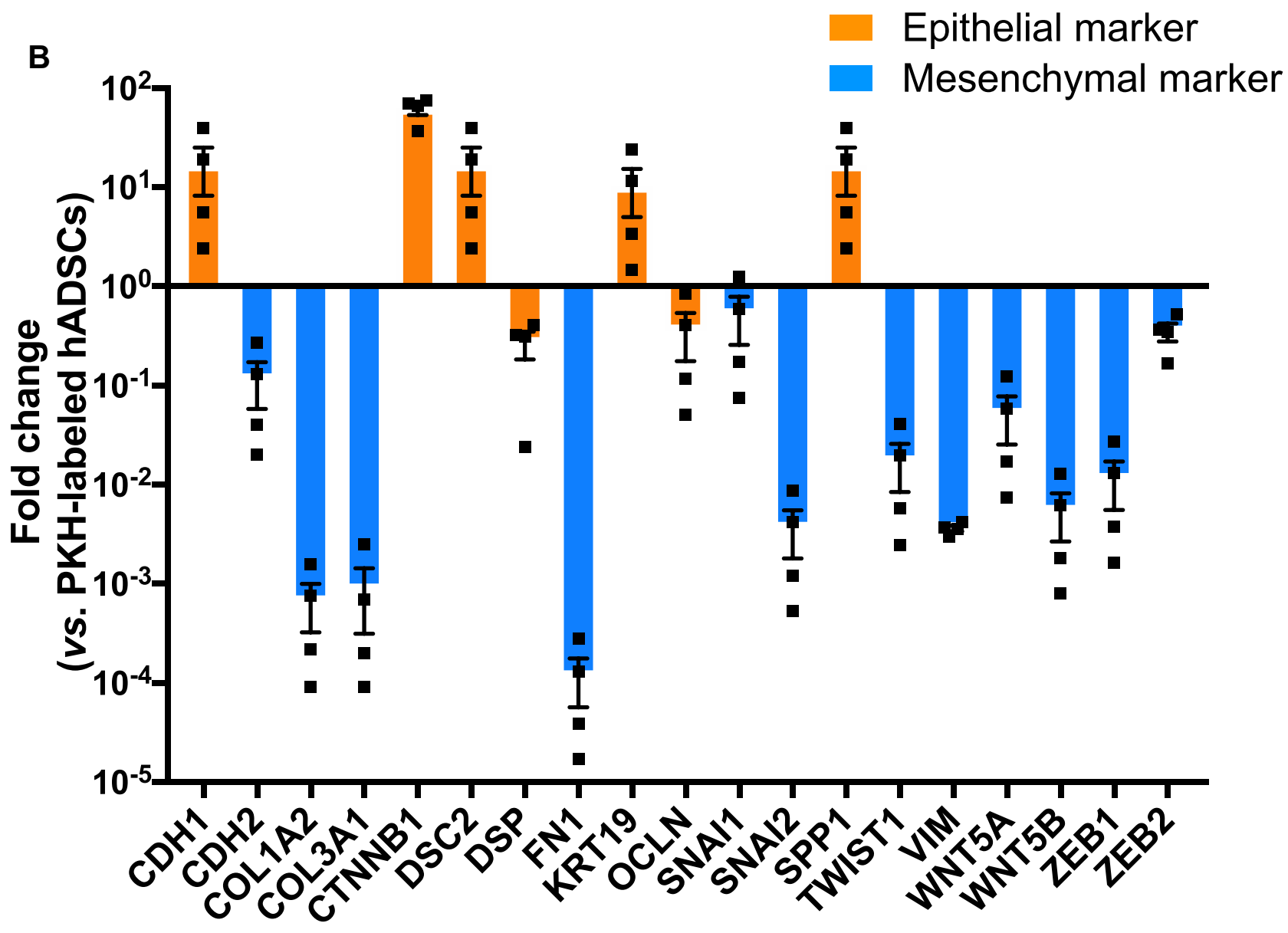

Figure 3 Gene expression of epithelial and mesenchymal genes in hADSCs isolated from lungs.

Notes: SCID-beige mice were intratracheally injected with 1.25 Uelastase and then intravenously injected with $1 \times 10^{6}$ PKH26-labeled hADSCs onday 3 . Onday 28 , lungs were collected for analyses. (A) PKH26-labeled hADSCs were identified and sorted from the lungs of the Elastase-hADSC group (Elastase i.t. + hADSCs i.v.). (B) Gene expression changes in hADSCs isolated from the lungs of the Elastase-hADSC group $(n=4)$ compared with naive $h A D S C s(n=4)$ using $R T^{2 \Theta}$ profiler ${ }^{T M}$ PCR array human epithelial-to-mesenchymal transition. Relative values to the average of naïv hADSCs are presented as means \pm SEM with individual plots.

Abbreviations: hADSCs, human adipose-derived mesenchymal stem cells; SEM, standard error of the mean. 
A

Saline i.t.

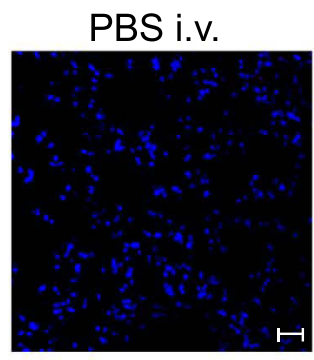

hADSCs i.v.
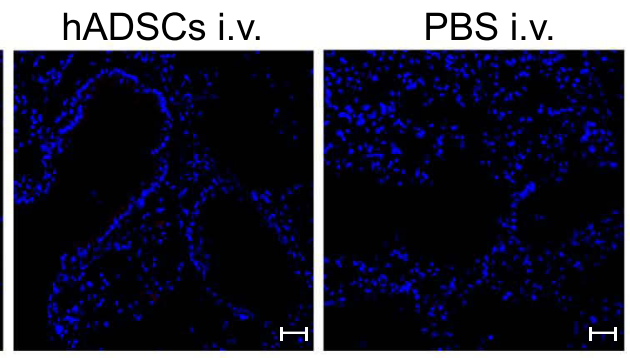

Elastase i.t.

B

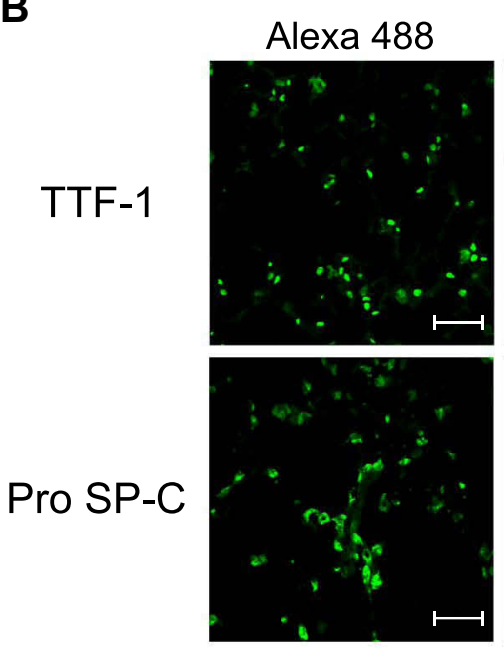

$\mathrm{PKH}-26$

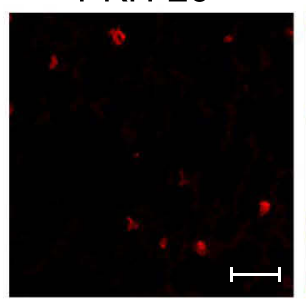

Merge+DAPI
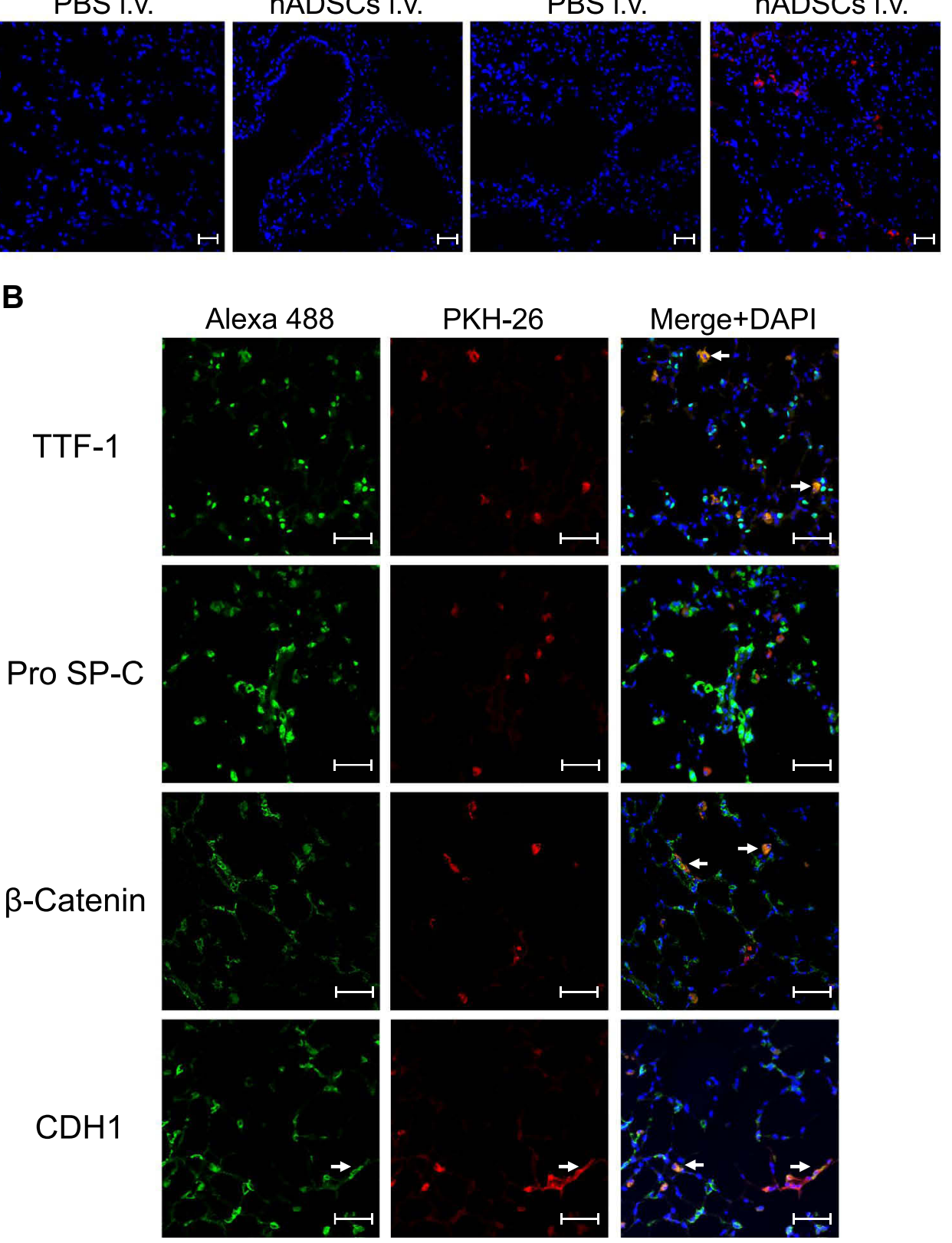

Figure 4 Fluorescence staining of epithelial markers in the lungs.

Notes: Lung sections from SCID-beige mice administered intravenously with PKH26-labeled hADSCs were observed under aconfocal laser microscope. (A) Fluorescence of PKH-26 (red) was observed with nuclear counterstaining by DAPI (blue) $(\times 200)$. (B) Expression of epithelial cells markers TTF-I, Pro SP-C, $\beta-$ Catenin, and CDHI visualized by Alexa Fluor 488 (green) in PKH26-labeled hADSCs (red) ( $\times 400)$. Arrow shows Alexa Flour 488 and PKH-26 merged cells. Scale bar shows $50 \mu$ m. Data are representative of more than three independent experiments.

Abbreviations: hADSCs, human adipose-derived mesenchymal stem cells; DAPI, 4',6-diamidino-2-phenylindole; TTF-I, thyroid transcription factor-I; SP-C, surfactant protein C; CDHI, cadherin-I.

pulmonary diseases including COPD have reported the effects of MSCs including BM-MSCs and ADSCs. ${ }^{11-}$ 16,21,30-36 Several clinical trials in COPD patients have shown that administration of BM-MSCs or ADSCs is safe, but there is no report showing therapeutic effects such as improved the pathogenesis and lung functions using human ADSCs. Therefore, we here focused on the effects of hADSCs for COPD. And it has been reported that human non-autologous stem cells may be immunogenic in animal models, so we used SCID-beige mice as the recipient experimental animals in this study. ${ }^{37}$ Emphysema is a characteristic histological change in 
COPD, and one widely used animal model of emphysema is the elastase treatment. In this study, systemic administration of hADSCs resulted in histological changes in an elastase-induced lung emphysema model mouse. Also, COPD causes not only emphysematous changes in the lungs but also airflow obstruction. In humans, in respiratory function tests, the ratio of forced expiratory volume in one second $\left(\mathrm{FEV}_{1}\right)$ to forced vital capacity (FVC) of less than 0.7 after administration of bronchodilators in respiratory function tests is essential for diagnosis of COPD. ${ }^{38}$ Recently, it has become possible to evaluate respiratory function in mice using the Forced Oscillation Technique, ${ }^{39}$ and its usefulness has been reported in mouse models of respiratory diseases. ${ }^{23,40-43}$ So we evaluated the effects of hADSCs on respiratory function using flexiVent ${ }^{\circledR}$ in a mouse model of elastase-induced emphysema. It has been reported that forced expiratory volume at $0.1 \mathrm{~s}$ $\left(\mathrm{FEV}_{0.1}\right)$, forced vital capacity (FVC), forced expiratory flow at $0.1 \mathrm{~s}\left(\mathrm{FEF}_{0.1}\right)$, inspiratory capacity (IC), Newtonian resistance $(\mathrm{Rn})$, and tissue hysteresivity $(\mathrm{G} / \mathrm{H})$ increase and $\mathrm{FEV}_{0.1} / \mathrm{FVC}$, peak expiratory flow (PEF), tissue damping $(\mathrm{G})$, and tissue elastance $(\mathrm{H})$ decrease in lung functions of a mouse model of emphysema established by intranasal administration of porcine pancreatic elastase three times a week to male $\mathrm{BALB} / \mathrm{c}$ mice. ${ }^{23}$ In another model of emphysema induced by intratracheal administration of porcine pancreatic elastase to $\mathrm{BALB} / \mathrm{c}$ mice, it has been reported that IC and Rrs (respiratory system resistance) increased and $\mathrm{H}$ decreased. ${ }^{44}$ We found that $\mathrm{FEV}_{0.1}$ was significantly decreased in the mice of elastase-induced emphysema as reported previously, ${ }^{23}$ and that systemic administration of hADSCs to the mice of elastaseinduced emphysema significantly improved the decrease in $\mathrm{FEV}_{0.1}$. This indicates that hADSCs not only improve COPD histologically, but also improve pulmonary function. On the other hand, systemic administration of hADSCs to elastase-induced COPD model mice did not improve FVC, $\mathrm{FEV}_{0.1} / \mathrm{FVC}$, Cst, $\mathrm{FEF}_{0.1}, \mathrm{PEF}, \mathrm{Rn}, \mathrm{G}, \mathrm{H}$, $\mathrm{IC}$, and Rrs in this study. Compared with a previous report, ${ }^{23,44}$ the difference of elastase administered to induce emphysema and that of respiratory function measurement method, flexiVent ${ }^{\circledR}$ and Buxco lung function analysis, might result in no significant difference in lung compliance or resistance.

In addition, hADSCs were detected in the lung parenchyma 25 days after systemic administration of hADSCs in elastase-induced emphysema mice. Previous reports have shown that systemically administered ADSCs were detected in the lung parenchyma and airways from day 1 to 21 after administration. ${ }^{15}$ Intravenously administered hADSCs migrated into the lungs of the Elastase-hADSC group, while very few hADSCs could be detected in the lungs of the hADSC group (Saline i.t. + hADSCs i.v.), which suggests that intravenously administered hADSCs are recruited to the site of lung injury. It has been proposed that various chemokines and cytokines are involved in the mechanism of stem cell recruitment to the site of injury. ${ }^{45}$ However, there are no reports on stem cell homing to emphysematous lungs, which requires further investigation.

We found that hADSCs attracted to emphysematous lungs led to downregulate mesenchymal-related gene expression, while epithelial-related gene expression was upregulated, which suggests induction of mesenchymalepithelial transition. Setiawan et al reported that inhibition of GSK3 and TGF- $\beta$ signaling causes human ADSCs to differentiate into epithelial cells through mesenchymalepithelial transition with downregulation of mesenchymal genes (Snail, Zeb1, and Cdh2) and upregulation of epithelial progenitor genes (E-cadherin, $\delta \mathrm{Np} 63$, Cytokeratins, and Occludin). ${ }^{46}$ Additionally, it has been reported that $\mathrm{Wnt} / \beta$-catenin signaling is inactivated by COPD and its reactivation restores the structure and function of the alveolar epithelium and improves bronchial expansion in an emphysema model. In addition, Wnt5A, which is a ligand that mediates $\beta$-catenin-independent Wnt signaling, ${ }^{47}$ exacerbates bronchial enlargement in elastase and smoking-induced emphysema models, whereas inhibition of Wnt5A reduces destruction of lung tissue and improves lung functions. This leads to re-epithelialization of alveoli and reactivation of $\mathrm{Wnt} / \beta$-catenin, ${ }^{48}$ which suggests that it might be a potential target for COPD treatment. ${ }^{49}$ Similarly, in this study, gene expression of $\beta$ catenin was up-regulated and that of Wnt5A was downregulated in hADSCs migrated to the emphysematous lungs, suggesting that hADSCs may have ameliorated emphysema by reactivating $\mathrm{Wnt} / \beta$-catenin signaling and inhibiting Wnt5A signaling.

Moreover, hADSCs attracted to emphysematous lungs expressed cell surface markers of type I alveolar epithelial cells, suggesting that hADSCs improved COPD by differentiating into type I alveolar epithelial cells. Previous studies reported that mouse BM-MSCs also differentiated into type I alveolar epithelial cells in elastase-induced emphysema, ${ }^{14}$ while mouse ADSCs differentiated into type II alveolar epithelial cells. ${ }^{21}$ As type II alveolar 
epithelial cells are progenitors of type I alveolar epithelial cells, ${ }^{50-52}$ it is possible that human ADSCs differentiated into type II alveolar epithelial cells and then into type I alveolar epithelial cells. The long time between administration of ADSCs and the analysis of ADSCs might have influenced the results of this study, and further investigation for the time points of analysis following ADSC administration is required.

Finally, we demonstrated the therapeutic effect of human-derived ADSCs on COPD using a COPD model established by administering elastase to SCID-beige mice, which suggests that hADSCs may be a valuable candidate for COPD medical therapy. But, the present study has still some limitations. For example, SCIDbeige mice were used in this study to exclude the effect of graft-versus-host disease, while we should consider the role of immunity in allograft hADSC administration for clinical trial. Second, because COPD in humans is mainly caused by smoking, our approach might also be valuable in other models of COPD, that have additional features of disease including airway/tissue inflammation such as cigarette smoke-induced models. Further studies are required to investigate whether the similar effects can be obtained from other models of COPD.

\section{Conclusion}

We found that hADSCs ameliorated emphysema both histologically and functionally, and migrated into the emphysematous lung in an elastase-induced COPD model in SCID-beige mice. The administered hADSCs into lungs underwent mesenchymal-epithelial transition in the emphysematous lungs and differentiated into type I alveolar epithelial cells. The results of this study may provide a mechanism for the treatment of COPD by ADSCs. Human ADSCs not only improved pulmonary function, but also had the potential to differentiate into alveolar epithelial cells in elastase-induced emphysema, which might be a source of fundamental therapy for COPD.

\section{Abbreviations}

ADSCs, adipose-derived mesenchymal stem cells; BMMSCs, bone marrow-derived MSCs; CDH1, cadherin-1; COPD, chronic obstructive pulmonary disease; Cst, static compliance; DAPI, 4',6-diamidino-2-phenylindole; $\mathrm{FEF}_{0.1}$, forced expiratory flow at $0.1 \mathrm{~s} ; \mathrm{FEV}_{0.1}$, forced expiratory volume at $0.1 \mathrm{~s} ; \mathrm{FEV}_{1}$, forced expiratory volume in one second; FVC, forced vital capacity; G, tissue damping;
$\mathrm{H}$, tissue elastance; hADSCs, human ADSCs; HLA, human leukocyte antigen; IC, inspiratory capacity; i.t., intratracheal; i.v., intravenously; Lm, mean linear intercept; MSCs, mesenchymal stem cells; PBS, phosphatebuffered saline; PEF, peak expiratory flow; Rn, Newtonian resistance; Rrs, respiratory system resistance; SEM, standard error of the mean; SP-C, surfactant protein C; TGF- $\beta$, transforming growth factor beta; TTF- 1 , thyroid transcription factor-1.

\section{Acknowledgments}

We thank Ms. Reiko Masuda and Ms. Hisayo Nishikawa (Department of Immunology, Nara Medical University) for their assistance. We also thank Mitchell Arico from Edanz (https://jp.edanz.com/ac) for editing a draft of this manuscript.

\section{Author Contributions}

All authors made substantial contributions to conception and design, acquisition of data, or analysis and interpretation of data; took part in drafting the article or revising it critically for important intellectual content; agreed to submit to the current journal; gave final approval of the version to be published; and agree to be accountable for all aspects of the work.

\section{Funding}

This work was supported by ROHTO Pharmaceutical Co., Ltd., and JSPS KAKENHI Grant Numbers JP16H05310, JP17H06392, JP19K08661, and JP20K08573.The funders had no role in study design, data collection and analysis, decision to publish, or preparation of the manuscript.

\section{Disclosure}

The authors report no conflicts of interest in this work.

\section{References}

1. Lopez-Campos JL, Tan W, Soriano JB. Global burden of COPD. Respirology. 2016;21(1):14-23. doi:10.1111/resp.12660

2. Soriano JB, Abajobir AA, Abate $\mathrm{KH}$, et al. Global, regional, and national deaths, prevalence, disability-adjusted life years, and years lived with disability for chronic obstructive pulmonary disease and asthma, 1990-2015: a systematic analysis for the global burden of disease study 2015. Lancet Respir Med. 2017;5(9):691-706. doi:10.1016/S2213-2600(17)30293-X

3. Rabe KF, Watz H. Chronic obstructive pulmonary disease. Lancet. 2017;389(10082):1931-1940. doi:10.1016/S0140-6736(17)31222-9

4. Antunes MA, Lapa ESJR, Rocco PR. Mesenchymal stromal cell therapy in COPD: from bench to bedside. Int $J$ Chron Obstruct Pulmon Dis. 2017;12:3017-3027. doi:10.2147/COPD.S146671 
5. Kocher AA, Schuster MD, Szabolcs MJ, et al. Neovascularization of ischemic myocardium by human bone-marrow-derived angioblasts prevents cardiomyocyte apoptosis, reduces remodeling and improves cardiac function. Nat Med. 2001;7(4):430-436. doi:10.1038/86498

6. Orlic D, Kajstura J, Chimenti S, et al. Mobilized bone marrow cells repair the infarcted heart, improving function and survival. Proc Natl Acad Sci USA. 2001;98(18):10344-10349. doi:10.1073/pnas.181177898

7. Horwitz EM, Prockop DJ, Fitzpatrick LA, et al. Transplantability and therapeutic effects of bone marrow-derived mesenchymal cells in children with osteogenesis imperfecta. Nat Med. 1999;5 (3):309-313. doi:10.1038/6529

8. Zhao DC, Lei JX, Chen R, et al. Bone marrow-derived mesenchymal stem cells protect against experimental liver fibrosis in rats. World J Gastroenterol. 2005;11(22):3431-3440. doi:10.3748/wjg.v11.i22.3431

9. Matthay MA, Goolaerts A, Howard JP, Lee JW. Mesenchymal stem cells for acute lung injury: preclinical evidence. Crit Care Med. 2010;38(10 Suppl):S569-573. doi:10.1097/CCM.0b013e3181f1ff1d

10. Kumamoto M, Nishiwaki T, Matsuo N, Kimura H, Matsushima K. Minimally cultured bone marrow mesenchymal stem cells ameliorate fibrotic lung injury. Eur Respir J. 2009;34(3):740-748. doi:10.1183/ 09031936.00128508

11. Longhini-Dos-Santos N, Barbosa-de-Oliveira VA, Kozma RH, et al. Cell therapy with bone marrow mononuclear cells in elastase-induced pulmonary emphysema. Stem Cell Rev Rep. 2013;9(2):210-218. doi:10.1007/s12015-012-9419-y

12. Cruz FF, Antunes MA, Abreu SC, et al. Protective effects of bone marrow mononuclear cell therapy on lung and heart in an elastase-induced emphysema model. Respir Physiol Neurobiol. 2012;182(1):26-36. doi:10.1016/j.resp.2012.01.002

13. Shigemura N, Okumura M, Mizuno S, Imanishi Y, Nakamura T, Sawa Y. Autologous transplantation of adipose tissue-derived stromal cells ameliorates pulmonary emphysema. Am J Transplant. 2006;6 (11):2592-2600. doi:10.1111/j.1600-6143.2006.01522.x

14. Katsha AM, Ohkouchi S, Xin H, et al. Paracrine factors of multipotent stromal cells ameliorate lung injury in an elastase-induced emphysema model. Mol Ther. 2011;19(1):196-203. doi:10.1038/ mt.2010.192

15. Schweitzer KS, Johnstone BH, Garrison J, et al. Adipose stem cell treatment in mice attenuates lung and systemic injury induced by cigarette smoking. Am J Respir Crit Care Med. 2011;183 (2):215-225. doi:10.1164/rccm.201001-0126OC

16. Hong Y, Kim YS, Hong SH, Oh YM. Therapeutic effects of adipose-derived stem cells pretreated with pioglitazone in an emphysema mouse model. Exp Mol Med. 2016;48(10):e266. doi:10.1038/ emm.2016.93

17. Han I, Kwon BS, Park HK, Kim KS. Differentiation potential of mesenchymal stem cells is related to their intrinsic mechanical properties. Int Neurourol J. 2017;21(Suppl 1):S24-31. doi:10.5213/ inj.1734856.428

18. D'Agostino B, Sullo N, Siniscalco D, De Angelis A, Rossi F. Mesenchymal stem cell therapy for the treatment of chronic obstructive pulmonary disease. Expert Opin Biol Ther. 2010;10(5):681-687. doi:10.1517/14712591003610614

19. Antunes MA, Abreu SC, Cruz FF, et al. Effects of different mesenchymal stromal cell sources and delivery routes in experimental emphysema. Respir Res. 2014;15(1):118. doi:10.1186/s12931-014-0118-x

20. Strioga M, Viswanathan S, Darinskas A, Slaby O, Michalek J. Same or not the same? Comparison of adipose tissue-derived versus bone marrow-derived mesenchymal stem and stromal cells. Stem Cells Dev. 2012;21(14):2724-2752. doi:10.1089/scd.2011.0722

21. Fukui E, Funaki S, Kimura K, et al. Adipose tissue-derived stem cells have the ability to differentiate into alveolar epithelial cells and ameliorate lung injury caused by elastase-induced emphysema in mice. Stem Cells Int. 2019;2019:5179172. doi:10.1155/2019/5179172
22. Weiss DJ, Casaburi R, Flannery R, LeRoux-Williams M, Tashkin DP. A placebo-controlled, randomized trial of mesenchymal stem cells in COPD. Chest. 2013;143(6):1590-1598. doi:10.1378/chest.12-2094

23. Devos FC, Maaske A, Robichaud A, et al. Forced expiration measurements in mouse models of obstructive and restrictive lung diseases. Respir Res. 2017;18(1):123. doi:10.1186/s12931-017-0610-1

24. Tomoda K, Kubo K, Dairiki K, et al. Whey peptide-based enteral diet attenuated elastase-induced emphysema with increase in short chain fatty acids in mice. BMC Pulm Med. 2015;15:64. doi:10.1186/ s12890-015-0059-2

25. Ota C, Yamada M, Fujino N, et al. Histone deacetylase inhibitor restores surfactant protein-C expression in alveolar-epithelial type II cells and attenuates bleomycin-induced pulmonary fibrosis in vivo. Exp Lung Res. 2015;41(8):422-434. doi:10.3109/01902148.2015.1060275

26. Gimble JM, Katz AJ, Bunnell BA. Adipose-derived stem cells for regenerative medicine. Circ Res. 2007;100(9):1249-1260. doi:10.1161/01.RES.0000265074.83288.09

27. Fraser JK, Wulur I, Alfonso Z, Hedrick MH. Fat tissue: an underappreciated source of stem cells for biotechnology. Trends Biotechnol. 2006;24(4):150-154. doi:10.1016/j.tibtech.2006.01.010

28. Hass R, Kasper C, Böhm S, Jacobs R. Different populations and sources of human mesenchymal stem cells (MSC): a comparison of adult and neonatal tissue-derived MSC. Cell Commun Signal. 2011;9:12. doi:10.1186/1478-811X-9-12

29. Izadpanah R, Trygg C, Patel B, et al. Biologic properties of mesenchymal stem cells derived from bone marrow and adipose tissue. $J$ Cell Biochem. 2006;99(5):1285-1297. doi:10.1002/jcb.20904

30. Adachi Y, Oyaizu H, Taketani S, et al. Treatment and transfer of emphysema by a new bone marrow transplantation method from normal mice to Tsk mice and vice versa. Stem Cells. 2006;24 (9):2071-2077. doi:10.1634/stemcells.2005-0575

31. Weiss DJ, Bertoncello I, Borok Z, et al. Stem cells and cell therapies in lung biology and lung diseases. Proc Am Thorac Soc. 2011;8 (3):223-272. doi:10.1513/pats.201012-071DW

32. Foronjy RF, Majka SM. The potential for resident lung mesenchymal stem cells to promote functional tissue regeneration: understanding microenvironmental cues. Cells. 2012;1(4):874. doi:10.3390/cells10 40874

33. Zhao Y, Xu A, Xu Q, et al. Bone marrow mesenchymal stem cell transplantation for treatment of emphysemic rats. Int J Clin Exp Med. 2014;7(4):968-972.

34. Yuhgetsu H, Ohno Y, Funaguchi N, et al. Beneficial effects of autologous bone marrow mononuclear cell transplantation against elastase-induced emphysema in rabbits. Exp Lung Res. 2006;32 (9):413-426. doi:10.1080/01902140601047633

35. Zhen G, Liu H, Gu N, Zhang H, Xu Y, Zhang Z. Mesenchymal stem cells transplantation protects against rat pulmonary emphysema. Front Biosci. 2008;13:3415-3422. doi:10.2741/2936

36. Zhen G, Xue Z, Zhao J, et al. Mesenchymal stem cell transplantation increases expression of vascular endothelial growth factor in papain-induced emphysematous lungs and inhibits apoptosis of lung cells. Cytotherapy. 2010;12(5):605-614. doi:10.3109/146532410037 45888

37. Grinnemo KH, Mansson A, Dellgren G, et al. Xenoreactivity and engraftment of human mesenchymal stem cells transplanted into infarcted rat myocardium. $J$ Thorac Cardiovasc Surg. 2004;127 (5):1293-1300. doi:10.1016/j.jtcvs.2003.07.037

38. Vogelmeier CF, Criner GJ, Martinez FJ, et al. Global strategy for the diagnosis, management, and prevention of chronic obstructive lung disease 2017 report. GOLD executive summary. Am J Respir Crit Care Med. 2017;195(5):557-582. doi:10.1164/rccm.201701-0218PP

39. McGovern TK, Robichaud A, Fereydoonzad L, Schuessler TF, Martin JG. Evaluation of respiratory system mechanics in mice using the forced oscillation technique. J Vis Exp. 2013;75:e50172. 
40. DiGiovanni FA, Ellis R, Wattie J, Hirota JA, Southam DS, Inman MD. Concurrent dual allergen exposure and its effects on airway hyperresponsiveness, inflammation and remodeling in mice. Dis Model Mech. 2009;2(5-6):275-282. doi:10.1242/dmm.001859

41. Tam A, Bates JH, Churg A, Wright JL, Man SF, Sin DD. Sex-related differences in pulmonary function following 6 months of cigarette exposure: implications for sexual dimorphism in mild COPD. PLoS One. 2016;11(10):e0164835. doi:10.1371/journal.pone.0164835

42. Parameswaran H, Suki B. Assessing structure-function relations in mice using the forced oscillation technique and quantitative histology. Methods Mol Biol. 2017;1639:77-91.

43. Bonnardel E, Prevel R, Campagnac M, et al. Determination of reliable lung function parameters in intubated mice. Respir Res. 2019;20 (1):211. doi:10.1186/s12931-019-1177-9

44. Robichaud A, Fereydoonzad L, Limjunyawong N, et al. Automated full-range pressure-volume curves in mice and rats. J Appl Physio. 2017;123(4):746-756. doi:10.1152/japplphysiol.00856.2016

45. Son BR, Marquez-Curtis LA, Kucia M, et al. Migration of bone marrow and cord blood mesenchymal stem cells in vitro is regulated by stromal-derived factor-1-CXCR4 and hepatocyte growth factor-c-met axes and involves matrix metalloproteinases. Stem Cells. 2006;24(5):1254-1264. doi:10.1634/stemcells.2005-0271

46. Setiawan M, Tan XW, Goh TW, Hin-Fai Yam G, Mehta JS. Inhibiting glycogen synthase kinase-3 and transforming growth factor-beta signaling to promote epithelial transition of human adipose mesenchymal stem cells. Biochem Biophys Res Commun. 2017;490 (4):1381-1388. doi:10.1016/j.bbrc.2017.07.036
47. Li C, Bellusci S, Borok Z, Minoo P. Non-canonical WNT signalling in the lung. J Biochem. 2015;158(5):355-365. doi:10.1093/jb/mvv081

48. Baarsma HA, Skronska-Wasek W, Mutze K, et al. Noncanonical WNT-5A signaling impairs endogenous lung repair in COPD. J Exp Med. 2017;214(1):143-163. doi:10.1084/jem.20160 675

49. Shi J, Li F, Luo M, Wei J, Liu X. Distinct roles of wnt/beta-catenin signaling in the pathogenesis of chronic obstructive pulmonary disease and idiopathic pulmonary fibrosis. Mediators Inflamm. 2017; 2017:3520581. doi:10.1155/2017/3520581

50. Desai TJ, Brownfield DG, Krasnow MA. Alveolar progenitor and stem cells in lung development, renewal and cancer. Nature. 2014;507(7491):190-194. doi:10.1038/nature12930

51. Tamo L, Hibaoui Y, Kallol S, et al. Generation of an alveolar epithelial type II cell line from induced pluripotent stem cells. Am J Physiol Lung Cell Mol Physiol. 2018;315(6):L921-L932. doi:10. 1152/ajplung.00357.2017

52. Jain R, Barkauskas CE, Takeda N, et al. Plasticity of Hopx(+) type I alveolar cells to regenerate type II cells in the lung. Nat Commun. 2015;6:6727. doi:10.1038/ncomms7727

\section{Publish your work in this journal}

The International Journal of COPD is an international, peer-reviewed journal of therapeutics and pharmacology focusing on concise rapid reporting of clinical studies and reviews in COPD. Special focus is given to the pathophysiological processes underlying the disease, intervention programs, patient focused education, and self management protocols. This journal is indexed on PubMed Central, MedLine and CAS. The manuscript management system is completely online and includes a very quick and fair peer-review system, which is all easy to use. Visit http://www.dovepress.com/testimonials.php to read real quotes from published authors. 